\title{
A miniaturized dual-band bandpass filter using composite resonators with flexible frequency ratio
}

Pin Wen ${ }^{1,2,3}$, Zhewang Ma ${ }^{1 \mathrm{a})}$, Haiwen Liu ${ }^{3 \mathrm{~b})}$, Shuangshuang $\mathbf{Z h u}^{3}$, Baoping Ren ${ }^{1,2}$, Xiaolong Wang ${ }^{1}$, and Masataka Ohira ${ }^{1}$

${ }^{1}$ Graduate School of Science and Engineering, Saitama University, 255 Shimo-Okubo, Sakura-ku, Saitama 338-8570, Japan

${ }^{2}$ College of Information Engineering, East China Jiaotong University, Nanchang 330013, People's Republic of China

${ }^{3}$ School of Electronic and Information Engineering, Xi'an Jiaotong University, $X i$ 'an 710049, People's Republic of China

a)ma@ees.saitama-u.ac.jp

b)haiwen_liu@hotmail.com

\begin{abstract}
A miniaturized dual-band bandpass filter (DBPF) using a pair of composite resonators is proposed in this letter. The composite resonator consists of two vertical short-circuited microstrip lines and a short transversal microstrip line, and its resonant characteristics are analyzed by using the even- and odd-mode method. The even- and odd-mode of the resonator can be controlled individually readily, and hence the DBPF can be designed with a flexible frequency ratio. The DBPF has a compact size of $0.06 \lambda_{\mathrm{g}} \times 0.14 \lambda_{\mathrm{g}}$. To verify the design, a compact DBPF with 3-dB fractional bandwidths of $6 \%$ and $7 \%$ is designed and fabricated. The measured response is in good agreement with the simulated result.
\end{abstract}

Keywords: dual-band bandpass filter (DBPF), composite resonator, flexible frequency ratio

Classification: Microwave and millimeter-wave devices, circuits, and modules

\section{References}

[1] J. M. Yan, et al.: "Design of a fourth-order dual-band bandpass filter with independently controlled external and inter-resonator coupling," IEEE Microw. Wireless Compon. Lett. 25 (2015) 642 (DOI: 10.1109/LMWC.2015.2463095).

[2] J. L. Xu, et al.: "Compact dual-mode dual-band bandpass filter with wide stopband for WLAN applications," Electron. Lett. 51 (2015) 1372 (DOI: 10. 1049/el.2015.1913).

[3] B. Wu, et al.: "Compact dual-band filter using defected stepped impedance resonator," IEEE Microw. Wireless Compon. Lett. 18 (2008) 674 (DOI: 10. 1109/LMWC.2008.2003459). 
elex.14.20170022).

[5] W. Jiang, et al.: "Compact dual-band filter using open/short stub loaded stepped impedance resonators (OSLSIRs/SSLSIRs)," IEEE Microw. Wireless Compon. Lett. 26 (2016) 672 (DOI: 10.1109/LMWC.2016.2597179).

[6] Z. C. Zhang, et al.: "Compact dual-band bandpass filters using open-/shortcircuited stub-loaded $\lambda / 4$ resonators," IEEE Microw. Wireless Compon. Lett. 25 (2015) 657 (DOI: 10.1109/LMWC.2015.2463216).

[7] M. Hayati and L. Noori: "Compact dual-band bandpass filter with ultra wide stopband using open-loop resonator loaded by T-shaped and open stubs," IEICE Electron. Express 8 (2011) 1168 (DOI: 10.1587/elex.8.1168).

[8] C. Yiran, et al.: "Novel design of substrate integrated waveguide filter employing broadside-coupled complementary split ring resonators," IEICE Electron. Express 12 (2015) 20150188 (DOI: 10.1587/elex.12.20150188).

[9] K. Dong, et al.: "A compact millimeter-wave dual-band bandpass filter using substrate-integrated waveguide (SIW) dual-mode cavities," IEICE Trans. Electron. E99-C (2016) 761 (DOI: 10.1587/transele.E99.C.761).

[10] X. Wang, et al:: "A novel dual bandpass filter incorporating left-handed transmission line based dual-band resonator," IEICE Electron. Express 12 (2015) 20150797 (DOI: 10.1587/elex.12.20150797).

\section{Introduction}

To meet the growing requirement for multi-service in wireless communications, dual-band bandpass filters (DBPFs) having simple design, low cost, compact size and independent control of the frequency ratio of its dual-passbands are required. In recently years, a number of approaches to realize DBPFs had been proposed $[1,2$, $3,4,5,6,7,8]$. In [1], a multiple orders DBPF was presented with two types of resonators having different resonant frequencies but common input and output feed lines. A ring resonator and a pair of strong coupled I/O feeding structure were introduced to configure a DBPF with a reduced circuit size was proposed in [2]. DBPFs are also realized using stepped-impedance resonators (SIRs) [3, 4] and stubloaded resonators (SLRs) $[5,6,7]$, which have dual or multiple non-degenerate modes and easy control of the frequency ratio of the dual-passbands. Moreover, substrate integrated waveguide (SIW) [8, 9], composite right-/left-handed (CRLH) structures [10], and other novel types of resonators were also used to design DBPFs. Nevertheless, the independent controllable of dual-passband performance and a compact circuit size are still strongly demanded in the DBPFs design.

In this Letter, a miniaturized DBPF is analyzed and realized with two cascaded composite resonators, and a flexible frequency ratio of its dual-passband is easily achieved. Moreover, by introducing a mixed electric and magnetic coupling, a good frequency selectivity and out-band rejection performance of the DBPF is realized.

\section{Proposed composite resonator}

Fig. 1a depicts the transmission line mode of the proposed composite resonator. It consists of two short-circuited microstrip lines and a short transversal connecting line. The characteristic impedances of these lines are all the same, and are indicated by $Z$. The electrical lengths of these lines are indicated by $\theta_{1}, \theta_{2}$ and $2 \theta_{3}$, 


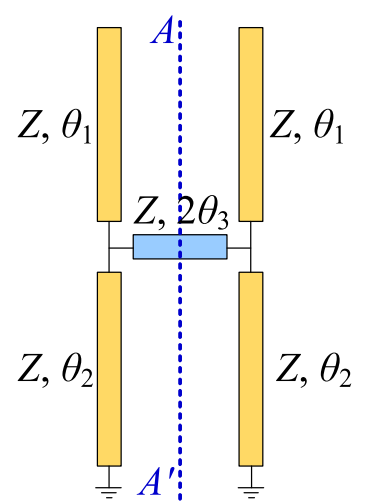

(a)

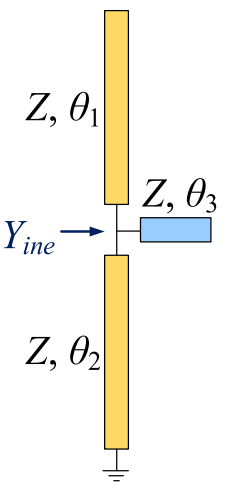

(b)

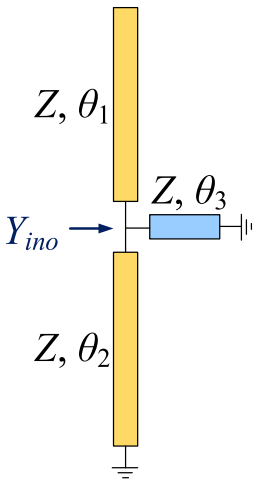

(c)

Fig. 1. (a) Transmission line model of the proposed composite resonator; (b) Even-mode equivalent circuit; (c) Odd-mode equivalent circuit.

respectively. Since the resonator is a symmetrical structure, the even- and oddmode method is good candidate for analyzing the proposed resonator. Under the even-/odd-mode excitation, the symmetrical plane $\mathrm{AA}^{\prime}$ behaves as a perfect magnetic wall or electric wall, respectively, and the even-/odd-mode equivalent circuit of the resonator are shown in Fig. $1 \mathrm{~b}$ and Fig. 1c, respectively. As illustrated in Fig. 1b, $Y_{\text {ine }}$ represent the input admittance under the even-mode excitation and is given by

$$
Y_{\text {ine }}=j Y_{0}\left(\tan \theta_{1}-\cot \theta_{2}+\tan \theta_{3}\right)
$$

At resonance, the input admittance $Y_{\text {ine }}=0$, thus the resonant condition is derived as

$$
\tan \theta_{1}-\cot \theta_{2}+\tan \theta_{3}=0
$$

Similarly, under the odd-mode excitation, the $Y_{\text {ino }}$ is express as

$$
Y_{\text {ino }}=j Y_{0}\left(\tan \theta_{1}-\cot \theta_{2}-\cot \theta_{3}\right)
$$

and the resonant condition is derived as

$$
\tan \theta_{1}-\cot \theta_{2}-\cot \theta_{3}=0
$$

Therefore, the resonant frequencies at the even-mode $\left(f_{\text {even }}\right)$ and odd-mode ( $\left.f_{\text {odd }}\right)$ can be calculated by solving (2) and (4). It can be seen from (2) that when $\theta 1$ is fixed and $\theta_{3}$ is very small compare with $\theta_{2}, \theta_{3}$ will has little effect on the evenmode frequency $f_{\text {even }}$. Under the same constraint, it can be seen from (4) that $\theta_{2}$ has less influence on the odd-mode frequency $f_{\text {odd }}$. Therefore, $f_{\text {even }}$ and $f_{\text {odd }}$, can be independently tuned by changing $\theta_{2}$ and $\theta_{3}$, respectively, with $\theta_{1}$ fixed.

To verify the analysis, EM simulation results of the resonator under a week coupling with $Z=98 \mathrm{Ohm}, \theta_{1}=54^{\circ}, \theta_{2}=36.7^{\circ}$, and $\theta_{3}=4^{\circ}$ are illustrated in Fig. 2a. The proposed composite resonator has two resonant modes, $f_{\text {even }}$ and $f_{\text {odd }}$. Moreover, two potential transmission zeros (TZs), $\mathrm{TZ}_{V G 1}$ and $\mathrm{TZ}_{V G 2}$, are observed at the lower and upper sides of two resonant modes, respectively, they are mainly produced by the virtual grounds existed in composite resonator (when $Y_{\text {ine }}$ and $Y_{\text {ino }}$ are equal to $\infty)$, and the phases reversal $\left(\Delta \phi=180^{\circ}\right)$ at the TZs. The variation of 


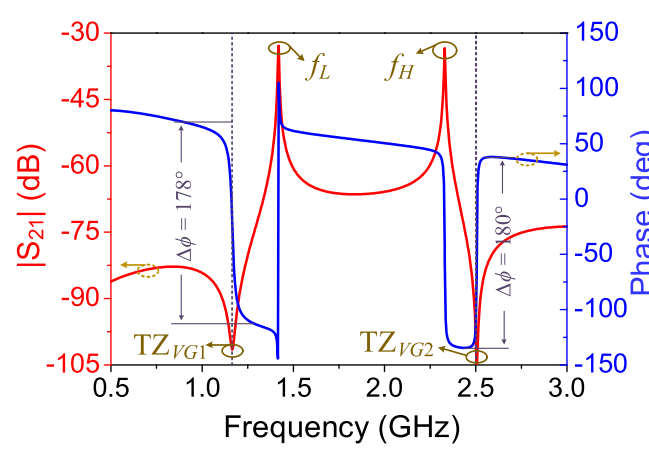

(a)

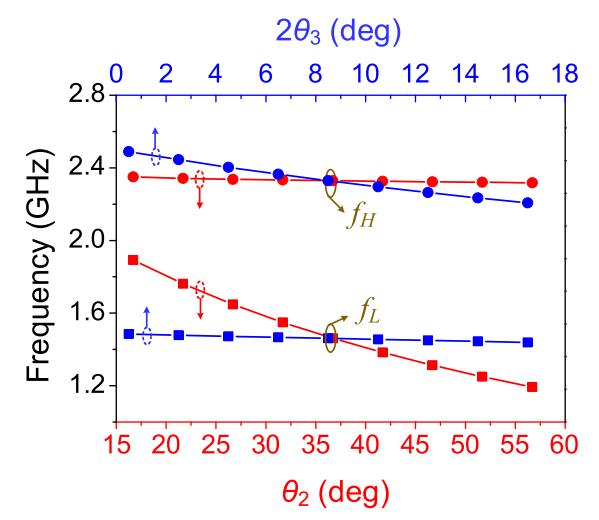

(b)

Fig. 2. (a) Simulated amplitude and phase of $S_{21}$, and (b) Variation of the even- and odd-mode resonant frequencies with $\theta_{2}$ and $2 \theta_{3}$.

resonant frequencies with $\theta_{2}$ and $2 \theta_{3}$ is illustrate in Fig. $2 \mathrm{~b}$, which verify that $f_{\text {even }}$ and $f_{\text {odd }}$ can be independently controlled by changing $\theta_{2}$ and $\theta_{3}$, respectively.

\section{Design of the dual-band bandpass filter}

The configuration of the DBPF is illustrated in Fig. 3. It consists of two composite resonators and a pair of side feeding lines. To realize the required coupling coefficients, the geometrical parameters $g_{1}, g_{i c}$, and $L_{i c}$ are determined by simulated extraction. On the other hand, the side feeding structures with dimension $L_{f 1}, L_{f 2}$, and $g_{0}$ are adjusted to meet the demanded external quality factors. Furthermore, for purpose of enhance the selectivity of the DBPF, the common ground via-hole and interdigital capacitor structures are introduced to form a mixed electric and magnetic coupling (MEMC), which, together with the inherent transmission zeros of the composite resonators, results in three transmission zeros $\left(\mathrm{TZ}_{1}, \mathrm{TZ}_{2}\right.$, and $\left.\mathrm{TZ}_{3}\right)$, as shown in Fig. 4.

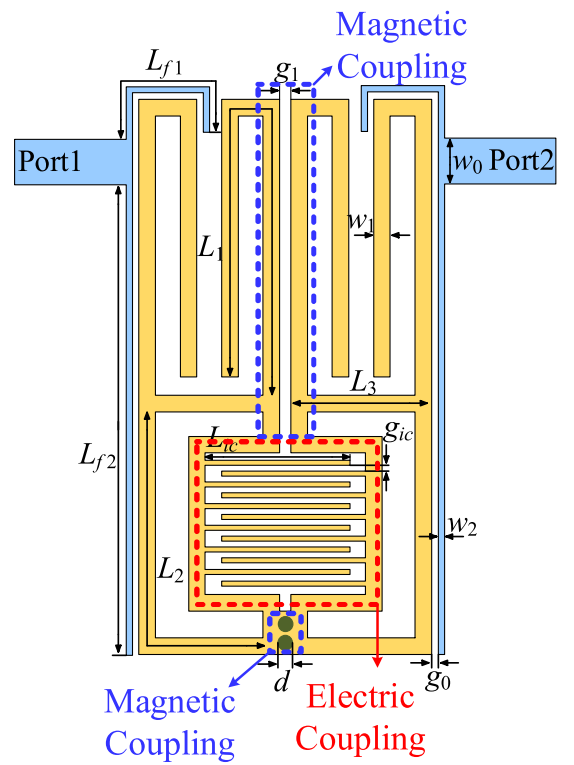

Fig. 3. Configuration of the proposed DBPF. 


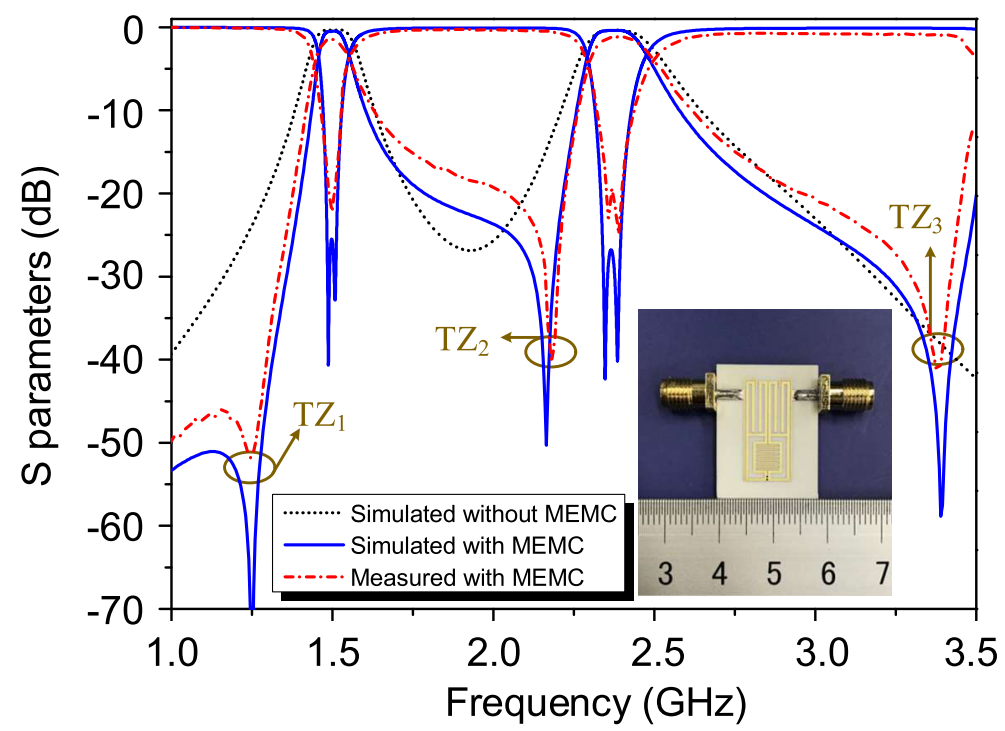

Fig. 4. Simulated and measured results and photograph of the fabricated DBPF.

\section{Results and discussion}

The DBPF is fabricated on Rogers RO $4003 \mathrm{C}$ substrate with a relative permittivity $\varepsilon_{r}=3.38$, loss tangent $\tan \delta=0.0027$, and a thickness $h=0.813 \mathrm{~mm}$, and the overall circuit size is $0.06 \lambda_{g} \times 0.14 \lambda_{g}$, where $\lambda_{g}$ is the guided wavelength at the center frequency of the lower passband. The final dimensions of the DBPF are: $L_{f 1}=6, L_{f 2}=15.2, g_{0}=0.2, L_{1}=20, L_{2}=13.5, L_{3}=3, L_{\mathrm{ic}}=3.95, w_{0}=1.8$, $w_{1}=0.5, w_{2}=0.2, g_{1}=0.15, g_{i c}=0.2$, and $d=0.4$ (Unit: $\mathrm{mm}$ ). The measured results and photograph of the DBPF are shown in Fig. 4. As shown in Fig. 4, the two passbands are centered at near $1.5 \mathrm{GHz}$ and $2.35 \mathrm{GHz}$, with a 3-dB fractional bandwidth of $5.96 \%$ and $7.19 \%$, respectively. The measured minimum in-band insertion losses are $1.43 \mathrm{~dB}$ and $1.15 \mathrm{~dB}$ at the two center frequencies, respectively. Both the return losses of the two passbands are greater than $20 \mathrm{~dB}$. Moreover, the three TZs are located at $1.25 \mathrm{GHz}, 2.19 \mathrm{GHz}$, and $3.39 \mathrm{GHz}$, respectively, which improve significantly the out-of-band suppression performance of the DBPF. Finally, Table I gives a comparison of the proposed DBPF with other reference works. It is seen that our DBPF has merits like low insertion loss and very compact circuit size.

Table I. Compared with previous DBPFs

\begin{tabular}{c|c|c|c|c|c|c}
\hline Ref. & $\begin{array}{c}\text { Center Freq. } \\
(\mathrm{GHz})\end{array}$ & $\begin{array}{c}\text { 3-dB FBWs } \\
(\%)\end{array}$ & $\begin{array}{c}\text { Insertion } \\
\text { Loss }(\mathrm{dB})\end{array}$ & $\begin{array}{c}\text { Return } \\
\text { Loss }(\mathrm{dB})\end{array}$ & $\begin{array}{c}\text { TPs/ } \\
\text { TZs }\end{array}$ & $\begin{array}{c}\text { Circuit Size } \\
\left(\lambda_{g} \times \lambda_{g}\right)\end{array}$ \\
\hline$[1]$ & $2.4 / 5.2$ & $6.4 / 5.8$ & $1.4 / 1.76$ & $15 / 19$ & $8 / 4$ & $0.21 \times 0.35$ \\
\hline$[2]$ & $2.4 / 5.2$ & $9.2 / 9.5$ & $1.2 / 2.7$ & $15 / 11$ & $4 / 4$ & $0.18 \times 0.18$ \\
\hline$[5]$ & $1.63 / 2.73$ & $7.5 / 5.1$ & $1.5 / 2.15$ & $17 / 23$ & $8 / 7$ & $0.25 \times 0.25$ \\
\hline$[6]$ & $2.4 / 5.8$ & $4.63 / 3.6$ & $1.35 / 1.97$ & $17 / 15$ & $4 / 2$ & $0.39 \times 0.25$ \\
\hline This work & $\mathbf{1 . 5 / 2 . 3 5}$ & $\mathbf{5 . 9 6 / 7 . 1 9}$ & $\mathbf{1 . 4 3 / 1 . 1 5}$ & $\mathbf{2 2 / 2 0}$ & $\mathbf{4 / 3}$ & $\mathbf{0 . 0 6} \times \mathbf{0 . 1 4}$ \\
\hline
\end{tabular}




\section{Conclusion}

A miniaturized dual-band bandpass filter using composite resonators with flexible frequency ratio is developed. Three transmission zeros are produced by introduced a mixed electric and magnetic coupling, which improves significantly the selectivity and out-band rejection performance. With the features of compactness, flexible design, and high performance, the proposed dual-band filter is attractive for dualband wireless communication applications, such as the GPS and the TD-LTE system.

\section{Acknowledgments}

This work was supported in part by the Grant-in-Aid for Scientific Research (KAKENHI 17K06373) from the Japan Society for the Promotion of Science, in part by the Young Science Foundation of Jiangxi Province of China (20171BAB212001), Yong Scientific Research Project of Jiangxi Province Education Department of China (GJJ160530), the National Science Foundation of China, (No. 61461020, U1431110), and the International Cooperation Funds of Jiangxi Province of China (No. 20151BDH80015). 